\title{
Characterization of Macrolide-Resistant Campylobacter coli Isolates from Food- Producing Animals on Farms Across Japan during 2004
}

\author{
Kazuki HARADA $^{1) *}$, Tetsuo ASAI ${ }^{1)}$, Akemi KOJIMA ${ }^{1)}$, Toshiya SAMESHIMA ${ }^{1)}$ and Toshio TAKAHASHI ${ }^{1)}$ \\ ${ }^{1)}$ National Veterinary Assay Laboratory, Ministry of Agriculture, Forestry and Fisheries, 1-15-1 Tokura, Kokubunji, Tokyo 185-8511, \\ Japan
}

(Received 7 March 2006/Accepted 8 June 2006)

ABSTRACT. We investigated the susceptibilities against 7 antimicrobial agents in Campylobacter jejuni and C. coli isolates from food-producing animals in 2004. In comparison with the results of past surveillance, no significant difference was observed in resistance rates against all of the antimicrobials tested in Campylobacter isolates. However, slight increase of erythromycin (EM) resistance was found in $C$. coli isolates from pigs. We examined the mutation of the $23 \mathrm{~S}$ rRNA gene and their susceptibilities against azithromycin, tylosin, and lincomycin in 44 EM-resistant isolates and 28 susceptible isolates of porcine origin. All the EM-resistant isolates contained A2075G in the 23S rRNA gene and showed cross-resistance to azithromycin, tylosin, and lyncomycin.

KEY WORDS: Campylobacter coli, erythromycin resistance, swine.

J. Vet. Med. Sci. 68(10): 1109-1111, 2006

Campylobacteriosis is among the most frequent types of bacterial food poisoning to occur in the Japanese population. Although campylobacteriosis is mainly caused by Campylobacter jejuni, C. coli is also recognized as an important food-borne pathogen [13]. Since erythromycin (EM) is the first-choice drug for the treatment of campylobacteriosis, infection with the EM-resistant Campylobacter spp. creates a risk of the failure of EM treatment. In this context, the emergence of EM resistance in Campylobacter isolates from food-producing animals poses a major problem for human medicine. In our previous study, we detected EM resistance in about half of the $C$. coli isolates examined but not in $C$. jejuni; resistance was mostly prevalent in the $C$. coli isolates from pigs on farms across Japan [7, 8].

It is known that mutations at positions 2074 and 2075 in the 23S rRNA gene are associated with EM resistance in the Campylobacter spp. [3-5]. However, there is little information available on the characteristics of the resistance mechanism of EM-resistant $C$. coli isolates from food-producing animals in Japan. Therefore, in the present study, we investigated the relationship between the mutation of the $23 \mathrm{~S}$ rRNA gene in EM-resistant $C$. coli strains very recently isolated from food-producing animals in Japan and their antimicrobial susceptibilities against lyncomycin and three agents belonging to the macrolide family of antibiotics.

A total of 215 Campylobacter isolates were obtained from 118 faecal samples of 118 apparently healthy animals (19 cattle, 42 pigs, 21 broiler chickens and 36 layer chickens) in 2004; these comprised 132 C. jejuni isolates (including 37,37 , and 58 isolates from cattle, broilers and layers, respectively) and $83 C$. coli isolates (including 72 and 11 from pigs and layers, respectively). Samples were taken

\footnotetext{
* Correspondence to: Harada, K., Animal Products Safety Division, Food Safety and Consumer Affairs Bureau, Ministry of Agriculture, Forestry and Fisheries, 1-2-1 Kasumigaseki, Chiyoda-ku, Tokyo 100-8950, Japan.
}

from one animal per farm in 11 prefectures (Miyagi, Nagano, Ibaraki, Chiba, Toyama, Osaka, Tottori, Yamaguchi, Kochi, Kumamoto and Okinawa prefectures). Isolation of Campylobacter was performed using modified cefoperazone charcoal deoxycholate (mCCDA) as previous described [8], and the identification of the isolates as $C$. jejuni or $C$. coli was carried out using multiplex polymerase chain reaction (PCR) [2]. C. jejuni and C. coli were isolated in 10 and all of the 11 prefectures tested, respectively. Antimicrobial susceptibility tests were carried out by an agar dilution method as previously described [8]. The distribution of minimum inhibitory concentrations (MICs) for each antimicrobial agent is shown in Table 1. In comparison with the results of past surveillance by the Japanese Veterinary Antimicrobial Resistance Monitoring Program conducted in 1999-2001 and 2002-2003 [7, 8], no significant difference was observed in resistance rates against all of the antimicrobials tested in Campylobacter isolates. However, slight increase of EM resistance in C. coli was found in the porcine isolates: $48.3 \%(70 / 145)$ in $1999-2001[7]$ and $48.5 \%(33 /$ 68 ) in $2002-2003$ [8] to $61.1 \%(44 / 72)$ in 2004 . Though EM resistance has been found in isolates from cattle $(100 \%$ $(3 / 3)$ in $1999-2001)$ and broiler chickens $(25.0 \%(1 / 4)$ in 1999-2001 and $16.7 \%(2 / 12)$ in 2002-2003) [7, 8], no isolate of $C$. coli was obtained in the present study. In layer chickens, EM resistance was not found in 2002-2003 $(0 \%$, $0 / 24$ ) [8] and in 2004, even though it was found in a small number of isolates $(40.0 \%, 2 / 5)[7]$. These results suggest that dynamics of EM resistance in porcine $C$. coli isolates should be noticed for a risk management of antimicrobial resistance in Campylobacter spp. PCR-restriction fragment length polymorphism [15] was applied for the detection of point mutations at positions 2074 (A2074C) and 2075 (A2075G) of the 23S rRNA gene in Campylobacter spp., which are homologous to positions 2058 and 2059 in Escherichia coli [15]. All of 44 EM-resistant (MIC $\geq 32$ 
Table 1. Distribution of MICs for a total of 215 Campylobacter isolates from food-producing animals in 2004

\begin{tabular}{|c|c|c|c|c|c|c|c|c|c|c|c|c|}
\hline \multirow{3}{*}{$\begin{array}{l}\text { Agents }^{\mathrm{a})} \\
\left(\mathrm{BP}^{\mathrm{b})}\right)\end{array}$} & \multicolumn{12}{|c|}{ Origins } \\
\hline & \multicolumn{3}{|c|}{ Cattle (37 of C. jejuni) } & \multicolumn{3}{|c|}{ Pigs (72 of C. coli) } & \multicolumn{3}{|c|}{ Broilers (37 of $C$. jejuni) } & \multicolumn{3}{|c|}{ Layers (58 of C. jejuni/ 11 of $C$. coli $)$} \\
\hline & $\mathrm{MIC}_{50}$ & $\mathrm{MIC}_{90}$ & $\begin{array}{c}\% \\
\text { Resistance }\end{array}$ & $\mathrm{MIC}_{50}$ & $\mathrm{MIC}_{90}$ & $\begin{array}{c}\% \\
\text { Resistance }\end{array}$ & $\mathrm{MIC}_{50}$ & $\mathrm{MIC}_{90}$ & $\begin{array}{c}\% \\
\text { Resistance }\end{array}$ & $\mathrm{MIC}_{50}$ & $\mathrm{MIC}_{90}$ & $\begin{array}{c}\% \\
\text { Resistance }\end{array}$ \\
\hline $\operatorname{ABPC}(32)$ & 2 & 16 & 8.1 & 4 & 8 & 8.3 & 4 & 16 & 8.1 & $4 / 8$ & $32 / 8$ & $17.2 / 9.1$ \\
\hline $\operatorname{DSM}(32)$ & 0.5 & 16 & 5.4 & 32 & $>512$ & 54.2 & 0.5 & 1 & 0 & $0.5 / 1$ & $1 / 1$ & $5.2 / 0$ \\
\hline GM & 0.25 & 1 & $-c)$ & 0.5 & 2 & - & 0.25 & 1 & - & $0.5 / 0.5$ & $0.5 / 0.5$ & - \\
\hline EM (32) & 1 & 1 & 0 & 128 & $>512$ & 61.1 & 1 & 4 & 0 & $1 / 1$ & $4 / 8$ & $0 / 0$ \\
\hline OTC (16) & 16 & 128 & 51.4 & 64 & 256 & 91.7 & 4 & 128 & 37.8 & $4 / 2$ & $128 / 128$ & $36.2 / 27.3$ \\
\hline NA (32) & 4 & 128 & 24.3 & 8 & 128 & 27.8 & 4 & 128 & 10.8 & $4 / 4$ & $128 / 64$ & $12.1 / 18.2$ \\
\hline ERFX (2) & $\leq 0.125$ & 4 & 16.2 & $\leq 0.125$ & 4 & 26.4 & $\leq 0.125$ & 2 & 10.8 & $\leq 0.125 / \leq 0.125$ & $4 / 4$ & $10.2 / 18.2$ \\
\hline
\end{tabular}

a) ABPC, ampicillin; DSM, dihydrostreptomycin; EM, erythromycin; OTC, oxytetracycline; NA, nalidixic acid; ERFX, enrofloxacin.

b) BP: Breakpoint ( $\mathrm{mg} / l)$ was defined by Ishihara et al. in $2006(8)$.

c) The breakpoint of gentamycin was not defined.

Table 2. Distribution of the MICs of macrolides and lyncomycin for 44 EM-resistant $C$. coli strains and 28 susceptible strains isolated from pigs in Japan

\begin{tabular}{|c|c|c|c|c|c|c|c|c|c|c|c|c|c|c|}
\hline \multirow{2}{*}{$\begin{array}{l}\text { Antimicrobial } \\
\text { agents }^{\text {a) }}\end{array}$} & \multirow{2}{*}{$\begin{array}{l}\text { 23S rRNA } \\
\text { gene } \\
\text { mutation }\end{array}$} & \multicolumn{12}{|c|}{$\mathrm{MIC}^{\mathrm{b})}(\mathrm{mg} / \mathrm{L})$} & \multirow[b]{2}{*}{$>512$} \\
\hline & & 0.25 & 0.5 & 1 & 2 & 4 & 8 & 16 & 32 & 64 & 128 & 256 & 512 & \\
\hline \multirow[t]{2}{*}{$\operatorname{EM}(14)^{c)}$} & $\mathrm{WT}^{\mathrm{d})}$ & 1 & 3 & 4 & 13 & 3 & 4 & & & & & & & \\
\hline & A2075G & & & & & & & & & & 2 & 3 & 17 & 22 \\
\hline \multirow[t]{2}{*}{ AZM (15) } & WT & 6 & 12 & 5 & 5 & & & & & & & & & \\
\hline & A2075G & & & & & & & & & & & & 2 & 42 \\
\hline \multirow[t]{2}{*}{ TS (16) } & WT & & & & 4 & 4 & 16 & & 4 & & & & & \\
\hline & A2075G & & & & & & & & & & 1 & & 9 & 34 \\
\hline \multirow[t]{2}{*}{ LCM } & WT & & & & & 5 & 5 & 11 & 6 & 1 & & & & \\
\hline & A2075G & & & & & & & & & & 1 & 7 & 12 & 24 \\
\hline
\end{tabular}

a) EM, erythromycin; AZM, azithromycin; TS, tylosin; LCM, lyncomycin.

b) MIC, minimum inhibitory concentration.

c) Numbers in the parenthesis means lacton ring numbers of macrolide antibiotics.

d) WT, wild type.

$\mathrm{mg} / \mathrm{L}$ ) isolates contained the $\mathrm{A} 2075 \mathrm{G}$ mutation but not the A2074C mutation in the 23S rDNA gene (Table 2). On the contrary, the $28 \mathrm{EM}$-susceptible isolates contained neither A2074C nor A2075G mutation. The A2075G mutation in the $23 \mathrm{~S}$ rRNA gene had previously been found in C. coli isolates from pigs in New Zealand [6], Denmark [9], France [12], and U.S.A. [14]. A similar mutation has been reported in EM-resistant C. coli isolates from patients in Japan [10]. Our previous study suggested that $C$. coli infection in humans is contracted from pigs [8]. Although infectious enteritis in humans caused by $C$. coli occurs infrequently in Japan [11], the prevalence of EM-resistance in $C$. coli should be continuously monitored both in public health and veterinary fields.

The A2075G mutation of the 23S rRNA gene may be the predominant resistance mechanism of EM-resistant $C$. coli isolates from pigs. Cagrielo et al. [1] have shown that EMresistant strains with the A2075G mutation exhibited crossresistance to 14-, 15-, and 16-member-ring macrolides. MICs of azithromycin, tylosin, and lincomycin for 72 por- cine $C$. coli isolates were determined as previously described [8]. As shown in Table 2, results similar to those of the previous report [1] were obtained, and the mutation was found to be involved in lincomycin resistance. In veterinary medicine, EM, tylosin, and lincomycin are considered important antimicrobial agents for the treatment of porcine diseases such as porcine mycoplasmosis, proliferative haemorrhagic enteropathy, and swine dysentery. However, the clinical use of macrolides and lincosamides may contribute to the increase in the EM-resistant $C$. coli strains and the maintenance of this resistance. Thus, the appropriate and prudent use of such antimicrobials is essential in the pig industry.

ACKNOWLEDGEMENTS. We are grateful to the staff of Livestock Hygiene Service Centers across Japan for collecting Campylobacter isolates from food-producing animals. This work was supported in part by grant-in aid of Ministry of Health, Labor and Welfare (H18-Shokuhin-Ippan-003). 


\section{REFERENCES}

1. Cagliero, C., Mouline, C., Payot, S. and Cloeckaert, A. 2005. J. Antimicrob. Chemother. 56: 948-950.

2. Cloak, O. M. and Fratamico, P. M. 2002. J. Food Prot. 65: 266-273.

3. Engberg, J., Aarestrup, F. M., Taylor, D. E., Gerner-Smidt, P. and Nachamkin, I. 2001. Emerg. Infect. Dis. 7: 24-34.

4. Gibreel, A., Kos, V. N., Keelan, M., Trieber, C. A., Levesque, S., Michaud, S. and Taylor, D. E. 2005. Antimicrob. Agent Chemother. 49: 2753-2759.

5. Haanperä, M., Huovinen, P. and Jalava, J. 2005. Antimicrob. Agent Chemother. 49: 457-460.

6. Harrow, S. A., Gilpin, B. J. and Klena, J. D. 2004. J. Appl. Microbiol. 97: 141-148.

7. Ishihara, K., Kira, T., Ogikubo, K., Morioka, A., Kojima, A., Kijima-Tanaka, M., Takahashi, T. and Tamura, Y. 2004. Int. J. Antimicrob. Agents 24: 63-69.

8. Ishihara, K., Yamamoto, T., Satake, S., Takayama, S., Kubota,
S., Negishi, H., Kojima, A., Asai, T., Sawada, T., Takahashi, T. and Tamura, Y. 2006. J. Appl. Microbiol. 100: 153-160.

9. Jensen, L. B. and Aarestrup, F. M. 2001. Antimicrob. Agent Chemother. 45: 371-372.

10. Niwa, H., Asai, Y., Yamai, S. and Itoh, K. 2004. Vet. Rec. 155: 395-396.

11. Obana, M., Sagara, H., Aoki, T., Kim, R., Takizawa, Y., Tsunoda, T., Irimajiri, S. and Yamashita, K. 2002. J. J. A. Inf. D. 76: 355-368 (in Japanese with English summary).

12. Payot, S., Avrain, L., Magras, C., Praud, K., Cloeckaert, A. and Chaslus-Dancla, E. 2004. Int. J. Antimicrob. Agents 23: 468472.

13. Tam, C. C., O’Brien, S. J., Adak, G. K., Meakins, S. M. and Frost, J. A. 2003. J. Infect. 47: 28-32.

14. Thakur, S. and Gebreyes, W. A. 2005. J. Clin. Microbiol. 43: 5705-5714.

15. Vacher, S., Ménard, A., Bernard, E. and Mégraud, F. 2003. Antimicrob. Agent Chemother. 47: 1125-1128. 